\title{
Moscas-das-frutas (DIPTERA: TEPHRITIDAE), suas plantas hospedeiras e parasitoides (HYMENOPTERA: BRACONIDAE) no norte do estado de Mato Grosso, Brasil
}

\author{
Michele Emily Souza da SILVA ${ }^{1}$, Michel Ariquenes WOCHNER ${ }^{1}$, Maria do Socorro Miranda de SOUSA ${ }^{2}$, \\ Marliton Rocha BARRETO ${ }^{1 *}$, Ricardo ADAIME ${ }^{3}$
}

\author{
${ }^{1}$ Instituto de Ciências Naturais, Humanas e Sociais, Universidade Federal de Mato Grosso, Sinop, MT, Brasil. \\ ${ }^{2}$ Programa de Pós-graduação em Biodiversidade Tropical, Universidade Federal do Amapá, Macapá, AP, Brasil. \\ ${ }^{3}$ Embrapa Amapá, Macapá, AP, Brasil. \\ *E-mail: mrb.ufmt@gmail.com
}

Recebido em outubro/2018; Aceito em abril/2019.

\begin{abstract}
RESUMO: As moscas-das-frutas estão presentes em todas as regiões do Brasil e são responsáveis por grandes perdas econômicas em pomares comerciais. Este trabalho teve o objetivo de determinar a riqueza de espécies de moscas-das-frutas, suas respectivas plantas hospedeiras e seus parasitoides em municípios da região norte de Mato Grosso. Foram realizadas coletas de frutos de 34 espécies vegetais, de julho de 2016 a novembro de 2017. Os frutos coletados foram acondicionados em recipientes plásticos para observar a emergência das moscas-dasfrutas e parasitoides. Foram obtidos 2.709 espécimes de moscas-das-frutas (cinco espécies) e 179 espécimes de parasitoides (três espécies). Este trabalho apresenta os primeiros registros de hospedeiros de moscas-das-frutas para o Mato Grosso. Adicionalmente, os parasitoides Utetes anastrephae Viereck e Asobara anastrephae (Muesebeck) são relatados pela primeira vez no Estado.
\end{abstract}

Palavras-chave: Anastrepha; Ceratitis capitata; Relação tritrófica; Amazônia Meridional.

\section{Fruit flies, their host plants and parasitoids in the northern state of Mato Grosso, Brazil}

\begin{abstract}
Fruit flies are present in all regions of Brazil and are responsible for large economic losses in commercial orchards. This work aimed to determine the richness of fruit fly species, their host plants and their parasitoids in municipalities in the northern region of Mato Grosso. We collected fruits from 34 plant species, from July 2016 to November 2017. The collected fruits were packed in plastic containers to observe the emergence of fruit flies and parasitoids. We obtained 2,709 specimens of fruit flies (five species) and 179 specimens of parasitoids (three species). This work presents the first records of hosts of fruit flies for Mato Grosso. In addition, the parasitoids Utetes anastrephae Viereck and Asobara anastrephae (Muesebeck) are reported for the first time in the State.
\end{abstract}

Keywords: Anastrepha; Ceratitis capitata; Tritrophic relationship; Southern Amazon.

\section{INTRODUÇÃO}

Presente em todos os estados brasileiros, a fruticultura é responsável pela geração direta de milhões de empregos em todo o ciclo produtivo e em diversas funções. Álvares; Bayma (2017) relataram que em 2014 a produção de frutas superou 39 milhões de toneladas e apresentou valor bruto total de $\mathrm{R} \$ 25,4$ bilhões. Segundo esses mesmos autores, a Amazônia Legal, em 2014, respondeu por 7,34\% do total de frutas produzidas no Brasil e proporcionou uma receita de $\mathrm{R} \$ 2,7$ bilhões para uma produção de 2.928.040 toneladas de frutas, obtidas em área superior a 226 mil hectares.

As moscas-das-frutas (Diptera: Tephritidae) estão entre as principais pragas agrícolas mundiais por causarem impacto econômico direto, decorrente da oviposição das fêmeas e alimentação das larvas, tornando os frutos não comercializáveis, além das restrições quarentenárias impostas por países importadores para evitar a entrada dessas pragas em seus territórios (ALUJA; MANGAN, 2008). Esse grupo de insetos têm sido o maior motivo de preocupação nos pomares ao redor do mundo. As perdas anuais chegam a 2 bilhões de dólares e, somente no Brasil, alcançam aproximadamente 200 milhões de dólares, envolvendo prejuízos na produção e comercialização e custos alfandegários com o comércio internacional, tornando o controle efetivo das moscas-dasfrutas uma necessidade global (CAMARGOS et al., 2017).

Há que considerar que menos de $0,5 \%$ das mais de 5.000 espécies de Tephritidae descritas são consideradas pragas de importância econômica (ALUJA et al., 2014). Esses insetos têm sido pouco estudados em áreas florestais com vegetação nativa. Logo, estudos envolvendo moscas-das-frutas precisam ser intensificados nessas áreas objetivando a obtenção de informações que permitam a identificação de espécies, compreensão de suas relações com hospedeiros nativos e/ou silvestres, bem como conhecimento das interações tritróficas entre tefritídeos silvestres, plantas hospedeiras e parasitoides associados nesses ambientes (JESUS-BARROS et al., 2012).

Com os esforços da pesquisa científica realizados nos anos recentes, hoje sabe-se que na Amazônia Legal estão assinaladas 78 espécies de moscas-das-frutas do gênero Anastrepha. Anastrepha striata Schiner e A. obliqua (Macquart) são as mais polífagas, apresentando ampla distribuição na região. Amazonas e Amapá são os Estados com o maior número de espécies registradas, 42 e 37 , respectivamente (ADAIME et al., 2016).

Os parasitoides (Hymenoptera) têm se destacado como os mais efetivos inimigos naturais das moscas-das-frutas 
(OVRUSKI et al., 2000; SILVA et al., 2013), especialmente os pertencentes à família Braconidae (subfamília Opiinae). Doryctobracon areolatus (Szépligeti) é uma das espécies de parasitoides de tefritídeos mais comuns e amplamente distribuídas, ocorrendo da Argentina até o sul dos Estados Unidos (OVRUSKI et al., 2000). Na Amazônia brasileira estão assinaladas oito espécies de Braconidae, com a predominância de D. areolatus e Opius bellus Gahan. Também ocorrem três espécies de Figitidae, predominando Aganaspis pelleranoi (Brèthes) (ADAIME et al., 2018).

A fruticultura se constitui atividade de grande importância social e econômica, através da geração de empregos e oportunidades no estado do Mato Grosso, que apresenta mais de 4 mil estabelecimentos produtores distribuídos entre horticultura e fruticultura, totalizando área de aproximadamente 687.666 hectares (IBGE, 2006). A fruticultura, com uma cesta de 17 produtos associados à Agricultura Familiar, produziu 191.307 toneladas em 2015, em estabelecimentos rurais de 113 municípios (PEAF MT, 2017).

Diante do potencial de danos ocasionados pelas moscasdas-frutas e dos riscos que as espécies-praga representam para a fruticultura em âmbito mundial, é de extrema importância o conhecimento das espécies presentes no estado do Mato Grosso, não somente em áreas produtoras, mas também as que possuem potencial produtivo.

\section{MATERIAL E MÉTODOS}

\section{1. Área de estudo}

O presente trabalho foi realizado nos municípios de Guarita, Sinop e Terra Nova do Norte, estado do Mato Grosso. O clima da região, de acordo com a classificação de Köppen, adequa-se ao tipo Aw, que é caracterizado por estações seca e chuvosa bem definidas, média pluviométrica anual de $2.000 \mathrm{~mm}$ e altas temperaturas, com média anual de $30{ }^{\circ} \mathrm{C}$ (Celsius), tendo $15^{\circ} \mathrm{C}$ de diferença entre o mês mais quente e o mês mais frio (ALVARES et al., 2013).

\subsection{Amostragem de frutos}

Foram coletadas amostras de frutos potencialmente infestados em pomares de sítios, comunidades e em fundos de quintais, no período de julho de 2016 a novembro de 2017 . Os frutos foram recolhidos tanto das árvores quanto do solo e posteriormente armazenados em sacos de papel tipo kraft, para transporte até o Laboratório de Entomologia da Universidade Federal de Mato Grosso (UFMT), Campus Sinop. Em todos os pontos de coleta foram registradas as coordenadas geográficas, obtidas com auxílio de GPS (Global Positioning System) (Tabela 1).

Em laboratório, os frutos de cada amostra foram acondicionados agrupadamente em recipientes plásticos com tampas adaptadas com furos e vedação confeccionada com microtelas de tecido, para possibilitar a circulação de ar e impedir a saída dos insetos após a emergência. Como substrato para obtenção das pupas foram colocados de três a quatro centímetros de vermiculita expandida. Os recipientes foram acondicionados em estantes apropriadas e mantidos em temperatura ambiente, por cerca de 20 a 30 dias, período suficiente para emergência dos insetos. Para a coleta após a emergência dos adultos, os recipientes foram colocados em

ambiente frio (temperatura média aproximada de $-6{ }^{\circ} \mathrm{C}$ ) por cinco a dez minutos, visando a redução de movimentos dos insetos e para não afetar as pupas. Os insetos que emergiram (moscas e parasitoides) foram mortos e acondicionados em microtubos contendo álcool $70 \%$, devidamente etiquetados, para posterior identificação.

As espécies vegetais amostradas foram identificadas por comparação com exsicatas do Herbário CNMT (herbário participante do INCT - Herbário Virtual da flora e dos fungos) do Acervo Biológico da Amazônia Meridional (ABAM) da Universidade Federal de Mato Grosso, por meio de comparações com exsicatas e literatura específica (LORENZI et al., 2006).

\subsection{Identificação dos insetos}

Os exemplares de Anastrepha foram identificados com auxílio da chave dicotômica ilustrada de Zucchi et al. (2011). A identificação foi baseada na análise da genitália das fêmeas, por meio do exame dos acúleos extrovertidos, com auxílio de estereomicroscópio e microscópio óptico (40x). Além disso, também foram observadas outras características, como padrão alar, mesonoto, mediotergito e subescutelo. A identificação de Ceratitis capitata (Wiedemann) foi realizada com base em Zucchi (2015). Para a identificação dos parasitoides (Braconidae) foram utilizados os trabalhos de Canal; Zucchi, (2000) e Marinho et al. (2011).

\section{RESULTADOS}

Foram coletadas amostras de 34 espécies vegetais, agrupadas em 15 diferentes famílias botânicas (Tabela 1). Destas, 12 espécies foram registradas como plantas hospedeiras de moscas-das-frutas, pertencentes às famílias Anacardiaceae, Malpighiaceae, Myrtaceae e Oxalidaceae. Os frutos foram coletados de acordo com a disponibilidade em campo, o que influenciou na diversidade de espécies vegetais amostradas em cada município. Myrtaceae foi a família com maior número de espécies amostradas (9), seguida por Anacardiaceae (4) e Rutaceae (4).

Das amostras coletadas, foram obtidos 2.709 espécimes de moscas-das-frutas (cinco espécies) e 179 espécimes de parasitoides (três espécies). Foram registradas cinco espécies de tefritídeos: Anastrepha fraterculus (Wiedemann), $A$. obliqua (Macquart), A. sororcula Zucchi, A. striata Schiner e C. capitata (Figura 1).

Neste estudo três espécies de parasitoides foram registradas: D. areolatus, Utetes anastrephae Viereck e Asobara anastrephae (Muesebeck), todas obtidas no município de Nova Guarita (Figura 1). Em Sinop, somente $D$. areolatus e $U$. anastrephae foram obtidos (Tabela 3). Doryctobracon areolatus foi obtido de amostras de Averrhoa carambola (Oxalidaceae), Eugenia pyriformis (Myrtaceae), Plinia cauliflora (Myrtaceae), Psidium cattleianum (Myrtaceae), Psidium guajava (Myrtaceae) e Spondias purpurea (Anacardiaceae). Associação do parasitoide $D$. areolatus com $A$. striata em frutos de $P$. cattleianum e $P$. guajava, e com $C$. capitata em frutos de $A$. carambola foram verificadas (Tabela 3). O parasitoide $U$. anastrephae foi obtido por meio da coleta de frutos de A. carambola, E. pyriformis, $P$. cauliflora e $S$. purpurea. Foi verificada associação de $U$. anastrephae com A. obliqua em frutos de S. purpurea (Tabela 3). 
Tabela 1. Espécies vegetais amostradas e pontos de coleta dos frutos para obtenção de moscas-das-frutas e parasitoides em Cláudia, Gaúcha do Norte, Guarantã do Norte, Itaúba, Nova Guarita, Sinop e Terra Nova do Norte e suas respectivas coordenadas geográficas. (07/2016 a 11/2017).

Table 1. Species sampled and fruit collection points to obtain fruit flies and parasitoids in the municipalities of Cláudia, Gaúcha do Norte, Guarantã do Norte, Itaúba, Nova Guarita, Sinop and Terra Nova do Norte and their respective coordinates geographical. July 2016 to November 2017.

\begin{tabular}{|c|c|c|c|}
\hline Nomes científicos - Famílias & Nomes vernaculares & Localidade & Coordenadas \\
\hline Anacardium humile A.St.-Hil. - Anacardiaceae & Cajuzinho-do-cerrado & Itaúba I & \# Coordenada não registrada \\
\hline \multirow[t]{2}{*}{ Anacardium occidentale L.- Anacardiaceae } & Caju & Sinop I & $11^{\circ} 51^{\prime} 53.914^{\prime \prime} \mathrm{S}-55^{\circ} 29^{\prime} 0.812^{\prime \prime} \mathrm{O}$ \\
\hline & & Sinop IV & $11^{\circ} 51^{\prime} 35.759^{\prime \prime} \mathrm{S}-55^{\circ} 27^{\prime} 18.288^{\prime \prime} \mathrm{O}$ \\
\hline \multirow[t]{2}{*}{ Annona muricata L.- Annonaceae } & Graviola & Nova Guarita I & $10^{\circ} 18^{\prime} 21.863^{\prime \prime S}-55^{\circ} 24^{\prime} 9.968^{\prime \prime} \mathrm{O}$ \\
\hline & & Sinop III & $11^{\circ} 50^{\prime} 25.22^{\prime \prime} \mathrm{S}-55^{\circ} 31^{\prime} 48.49^{\prime \prime} \mathrm{O}$ \\
\hline Annona squamosa L.- Annonaceae & Fruta-do-conde & Sinop III & $11^{\circ} 50^{\prime} 25.22^{\prime \prime} \mathrm{S}-55^{\circ} 31^{\prime} 48.49^{\prime \prime} \mathrm{O}$ \\
\hline \multirow[t]{5}{*}{ Averrhoa carambola L.- Oxalidaceae } & Carambola & Cláudia & $11^{\circ} 30^{\prime} 26.5^{\prime \prime} \mathrm{S}-54^{\circ} 52^{\prime} 58.2^{\prime \prime O}$ \\
\hline & & Nova Guarita V & $10^{\circ} 18^{\prime} 22.388^{\prime \prime} \mathrm{S}-55^{\circ} 24^{\prime} 10.534^{\prime \prime} \mathrm{O}$ \\
\hline & & Nova Guarita IV & $10^{\circ} 18^{\prime} 21.355^{\prime \prime} \mathrm{S}-55^{\circ} 24^{\prime} 6.865^{\prime \prime} \mathrm{O}$ \\
\hline & & Sinop IX & $11^{\circ} 50^{\prime} 30.746^{\prime \prime} \mathrm{S}-55^{\circ} 32^{\prime} 1.442^{\prime \prime} \mathrm{O}$ \\
\hline & & Guarantã do Norte & $9^{\circ} 57^{\prime} 44.0^{\prime \prime} \mathrm{S}-54^{\circ} 533^{\prime} 24.8^{\prime \prime O}$ \\
\hline Carica papaya L..- Caricaceae & Mamão & Sinop XI & $11^{\circ} 51^{\prime} 34.736^{\prime \prime} \mathrm{S}-55^{\circ} 28^{\prime} 55.596^{\prime \prime} \mathrm{O}$ \\
\hline Citrus bergamia Risso et Poiteau - Rutaceae & Bergamota & Nova Guarita I & $10^{\circ} 18^{\prime} 21.863^{\prime \prime} \mathrm{S}-55^{\circ} 24^{\prime} 9.968^{\prime \prime} \mathrm{O}$ \\
\hline \multirow[t]{2}{*}{ Citrus latifolia Tanaka- Rutaceae } & Limão & Gaúcha do Norte & $13^{\circ} 6^{\prime} 59.173^{\prime \prime} \mathrm{S}-53^{\circ} 18^{\prime} 23.911^{\prime \prime} \mathrm{O}$ \\
\hline & & Sinop V & $11^{\circ} 51^{\prime} 45.81^{\prime \prime} \mathrm{S}-55^{\circ} 22^{\prime} 46.114^{\prime \prime} \mathrm{O}$ \\
\hline \multirow{3}{*}{ Citrus reticulata Blanco - Rutaceae } & Ponkan & Sinop & \# Coordenada não registrada \\
\hline & & Gaúcha do Norte & $13^{\circ} 6^{\prime} 59.173^{\prime \prime} \mathrm{S}-53^{\circ} 18^{\prime} 23.911^{\prime \prime} \mathrm{O}$ \\
\hline & & Nova Guarita I & $10^{\circ} 18^{\prime} 21.863^{\prime \prime} \mathrm{S}-55^{\circ} 24^{\prime} 9.968^{\prime \prime} \mathrm{O}$ \\
\hline \multirow[t]{2}{*}{ Citrus sinensis (L.), Osbeck - Rutaceae } & Laranja & Gaúcha do Norte & $13^{\circ} 6^{\prime} 59.173^{\prime \prime} \mathrm{S}-53^{\circ} 18^{\prime} 23.911^{\prime \prime} \mathrm{O}$ \\
\hline & & Nova Guarita I & $10^{\circ} 18^{\prime} 21.863^{\prime \prime} \mathrm{S}-55^{\circ} 24^{\prime} 9.968^{\prime \prime} \mathrm{O}$ \\
\hline Coffea arabica L. - Rubiaceae & Café & Cláudia & $11^{\circ} 31^{\prime} 20.615^{\prime \prime} \mathrm{S}-55^{\circ} 52^{\prime} 38.626^{\prime \prime} \mathrm{O}$ \\
\hline Syzygium jambos (L.) Alston - Myrtaceae & Jambo-amarelo & Sinop II & $11^{\circ} 52^{\prime} 24.002^{\prime \prime} \mathrm{S}-55^{\circ} 35^{\prime} 51.274^{\prime \prime} \mathrm{O}$ \\
\hline \multirow[t]{3}{*}{ Eugenia pyriformis Cambess - Myrtaceae } & Uvaia & Sinop V & $11^{\circ} 51^{\prime} 45.81^{\prime \prime} \mathrm{S}-55^{\circ} 22^{\prime} 46.114^{\prime \prime} \mathrm{O}$ \\
\hline & & Sinop XV & $11^{\circ} 51^{\prime} 56.887^{\prime \prime} \mathrm{S}-55^{\circ} 30^{\prime} 41.116^{\prime \prime} \mathrm{O}$ \\
\hline & & Itaúba II & $11^{\circ} 0^{\prime} 23.731^{\prime \prime} \mathrm{S}-55^{\circ} 14^{\prime} 25.541^{\prime \prime} \mathrm{O}$ \\
\hline Eugenia stipitata McVaugh - Myrtaceae & Araçá-boi & Nova Guarita I & $10^{\circ} 18^{\prime} 21.863^{\prime \prime} \mathrm{S}-55^{\circ} 24^{\prime} 9.968^{\prime \prime} \mathrm{O}$ \\
\hline \multirow{2}{*}{ Eugenia uniflora L.- Myrtaceae } & Pitanga & Sinop V & $11^{\circ} 51^{\prime} 45.81^{\prime \prime} \mathrm{S}-55^{\circ} 22^{\prime} 46.114^{\prime \prime} \mathrm{O}$ \\
\hline & & Itaúba II & $11^{\circ} 0^{\prime} 23.731^{\prime \prime} \mathrm{S}-55^{\circ} 14^{\prime} 25.541^{\prime \prime} \mathrm{O}$ \\
\hline \multirow[t]{2}{*}{ Ficus carica L.- Moraceae } & Figo & Nova Guarita II & $10^{\circ} 18^{\prime} 50.296^{\prime \prime} \mathrm{S}-55^{\circ} 24^{\prime} 33.455^{\prime \prime} \mathrm{O}$ \\
\hline & & Sinop IV & $11^{\circ} 51^{\prime} 35.759^{\prime \prime} \mathrm{S}-55^{\circ} 27^{\prime} 18.288^{\prime \prime} \mathrm{O}$ \\
\hline Genipa americana L.- Rubiaceae & Jenipapo & Sinop II & $11^{\circ} 52^{\prime} 24.002^{\prime \prime} \mathrm{S}-55^{\circ} 35^{\prime} 51.274^{\prime \prime} \mathrm{O}$ \\
\hline \multirow[t]{6}{*}{ Malpighia emarginata DC.- Malpighiaceae } & Acerola & Gaúcha do Norte & $13^{\circ} 6^{\prime} 59.173^{\prime \prime} \mathrm{S}-53^{\circ} 18^{\prime} 23.911^{\prime \prime} \mathrm{O}$ \\
\hline & & Nova Guarita I & $10^{\circ} 18^{\prime} 21.863^{\prime \prime} \mathrm{S}-55^{\circ} 24^{\prime} 9.968^{\prime \prime} \mathrm{O}$ \\
\hline & & Sinop V & $11^{\circ} 51^{\prime} 45.81^{\prime \prime} \mathrm{S}-55^{\circ} 22^{\prime} 46.114^{\prime \prime} \mathrm{O}$ \\
\hline & & Sinop XVI & $11^{\circ} 50^{\prime} 21.602^{\prime \prime} \mathrm{S}-55^{\circ} 29^{\prime} 54.481^{\prime \prime} \mathrm{O}$ \\
\hline & & Terra Nova do & $10^{\circ} 35^{\prime} 55.32^{\prime \prime} \mathrm{S}-55^{\circ} 6^{\prime} 53.798^{\prime \prime} \mathrm{O}$ \\
\hline & & Terra Nova do & $10^{\circ} 35^{\prime} 58.8^{\prime \prime} \mathrm{S}-55^{\circ} 07^{\prime} 08.3^{\prime \prime} \mathrm{O}$ \\
\hline \multirow[t]{3}{*}{ Mangifera indica L. - Anacardiaceae } & Manga & Sinop V & $11^{\circ} 51^{\prime} 45.81^{\prime \prime} \mathrm{S}-55^{\circ} 22^{\prime} 46.114^{\prime \prime} \mathrm{O}$ \\
\hline & & Sinop X & $11^{\circ} 50^{\prime} 21.466^{\prime \prime} \mathrm{S}-55^{\circ} 29^{\prime} 54.744^{\prime \prime} \mathrm{O}$ \\
\hline & & Nova Guarita I & $10^{\circ} 18^{\prime} 21.863^{\prime \prime} \mathrm{S}-55^{\circ} 24^{\prime} 9.968^{\prime \prime} \mathrm{O}$ \\
\hline Morinda citrifolia L.- Rubiaceae & Noni & Sinop XVII & $11^{\circ} 51^{\prime} 42.523^{\prime \prime} \mathrm{S}-55^{\circ} 30^{\prime} 8.626^{\prime \prime} \mathrm{O}$ \\
\hline \multirow[t]{3}{*}{ Morus nigra L.- Moraceae } & Amora & Sinop XVIII & $11^{\circ} 51^{\prime} 49.014^{\prime \prime} \mathrm{S}-55^{\circ} 28^{\prime} 59.354^{\prime \prime} \mathrm{O}$ \\
\hline & & Sinop II & $11^{\circ} 52^{\prime} 24.002^{\prime \prime} \mathrm{S}-55^{\circ} 35^{\prime} 51.274^{\prime \prime} \mathrm{O}$ \\
\hline & & Sinop I & $11^{\circ} 51^{\prime} 52.729^{\prime \prime} \mathrm{S}-55^{\circ} 29^{\prime} 0.017^{\prime \prime} \mathrm{O}$ \\
\hline Passiflora edulis Sims. - Passifloraceae & Maracujá & Sinop XIX & $11^{\circ} 51^{\prime} 50.886^{\prime \prime} \mathrm{S}-55^{\circ} 29^{\prime} 8.614^{\prime \prime} \mathrm{O}$ \\
\hline \multirow[t]{4}{*}{ Plinia cauliflora (Mart.) Kausel - Myrtaceae } & Jabuticaba & Nova Guarita I & $10^{\circ} 18^{\prime} 21.863^{\prime \prime} \mathrm{S}-55^{\circ} 24^{\prime} 9.968^{\prime \prime} \mathrm{O}$ \\
\hline & & Nova Guarita II & $10^{\circ} 18^{\prime} 50.296^{\prime \prime} \mathrm{S}-55^{\circ} 24^{\prime} 33.455^{\prime \prime} \mathrm{O}$ \\
\hline & & Sinop IV & $11^{\circ} 51^{\prime} 35.759^{\prime \prime} \mathrm{S}-55^{\circ} 27^{\prime} 18.288^{\prime \prime} \mathrm{O}$ \\
\hline & & Sinop V & $11^{\circ} 51^{\prime} 45.81^{\prime \prime} \mathrm{S}-55^{\circ} 22^{\prime} 46.114^{\prime \prime} \mathrm{O}$ \\
\hline Psidium cattleianum Afzel. ex Sabine - Myrtaceae & Araçá & Sinop V & $11^{\circ} 51^{\prime} 45.81^{\prime \prime} \mathrm{S}-55^{\circ} 22^{\prime} 46.114^{\prime \prime} \mathrm{O}$ \\
\hline Psidium guajava L.- Myrtaceae & Goiaba & Nova Guarita II & $10^{\circ} 18^{\prime} 50.296^{\prime \prime} \mathrm{S}-55^{\circ} 24^{\prime} 33.455^{\prime \prime} \mathrm{O}$ \\
\hline & & Sinop V & $11^{\circ} 51^{\prime} 45.81^{\prime \prime} \mathrm{S}-55^{\circ} 22^{\prime} 46.114^{\prime \prime} \mathrm{O}$ \\
\hline Punica granatum L.- Lythraceae & Romã & Sinop & $11^{\circ} 51^{\prime} 42.523^{\prime \prime} \mathrm{S}-55^{\circ} 30^{\prime} 8.626^{\prime \prime} \mathrm{O}$ \\
\hline & & Sinop IV & $11^{\circ} 51^{\prime} 35.759^{\prime \prime} \mathrm{S}-55^{\circ} 27^{\prime} 18.288^{\prime \prime} \mathrm{O}$ \\
\hline & & Nova Guarita II & $10^{\circ} 18^{\prime} 50.296^{\prime \prime} \mathrm{S}-55^{\circ} 24^{\prime} 33.455^{\prime \prime} \mathrm{O}$ \\
\hline Solanum lycocarpum A.St.-Hil. - Solanaceae & Lobeira & Sinop XII & $11^{\circ} 51^{\prime} 50.922^{\prime \prime} \mathrm{S}-55^{\circ} 29^{\prime} 0.128^{\prime \prime} \mathrm{O}$ \\
\hline Spondias purpurea L.- Anacardiaceae & Seriguela & Sinop II & $11^{\circ} 53^{\prime} 40.916^{\prime \prime} \mathrm{S}-55^{\circ} 38^{\prime} 36.78^{\prime \prime} \mathrm{O}$ \\
\hline & & Sinop X & $11^{\circ} 50^{\prime} 21.466^{\prime \prime} \mathrm{S}-55^{\circ} 29^{\prime} 54.744^{\prime \prime} \mathrm{O}$ \\
\hline & & Guarantã do Norte & $9^{\circ} 57^{\prime} 44.0^{\prime \prime} \mathrm{S}-54^{\circ} 53^{\prime} 24.8^{\prime \prime} \mathrm{O}$ \\
\hline Syzygium cumini (L.) Skeels - Myrtaceae & Jamelão & Sinop XIII & $11^{\circ} 51^{\prime} 43.441^{\prime \prime} \mathrm{S}-55^{\circ} 29^{\prime} 1.615^{\prime \prime} \mathrm{O}$ \\
\hline Syzygium malaccense (L.) Merr. \& L.M.Perry - Myrtaceae & Jambo-vermelho & Gaúcha do Norte & $13^{\circ} 6^{\prime} 59.173^{\prime \prime} \mathrm{S}-53^{\circ} 18^{\prime} 23.911^{\prime \prime} \mathrm{O}$ \\
\hline & & Sinop XIV & $11^{\circ} 50^{\prime} 8.484^{\prime \prime} \mathrm{S}-55^{\circ} 29^{\prime} 43.094^{\prime \prime} \mathrm{O}$ \\
\hline & & Sinop II & $11^{\circ} 52^{\prime} 24.002^{\prime \prime} \mathrm{S}-55^{\circ} 35^{\prime} 51.274^{\prime \prime} \mathrm{O}$ \\
\hline Talisia esculenta A.St.-Hil. - Sapindaceae & Pitomba & Sinop VIII & $11^{\circ} 51^{\prime} 30.406^{\prime \prime} \mathrm{S}-55^{\circ} 29^{\prime} 50.053^{\prime \prime} \mathrm{O}$ \\
\hline & & Sinop VII & $11^{\circ} 51^{\prime} 6.088^{\prime \prime} \mathrm{S}-55^{\circ} 31^{\prime} 12.587^{\prime \prime} \mathrm{O}$ \\
\hline Theobroma cacao L.- Malvaceae & Cacau & Cláudia & \# Coordenada não registrada \\
\hline Theobroma grandiflorum (Willd. ex Spreng.) - Malvaceae & Cupuaçu & Sinop VI & $11^{\circ} 50^{\prime} 03.5^{\prime \prime} \mathrm{S}-55^{\circ} 27^{\prime} 33.5^{\prime \prime} \mathrm{O}$ \\
\hline Thevetia peruviana (Pers.) K.Schum. - Apocynaceae & Chapéu-de-napoleão & Nova Guarita III & \# Coordenada não registrada \\
\hline
\end{tabular}




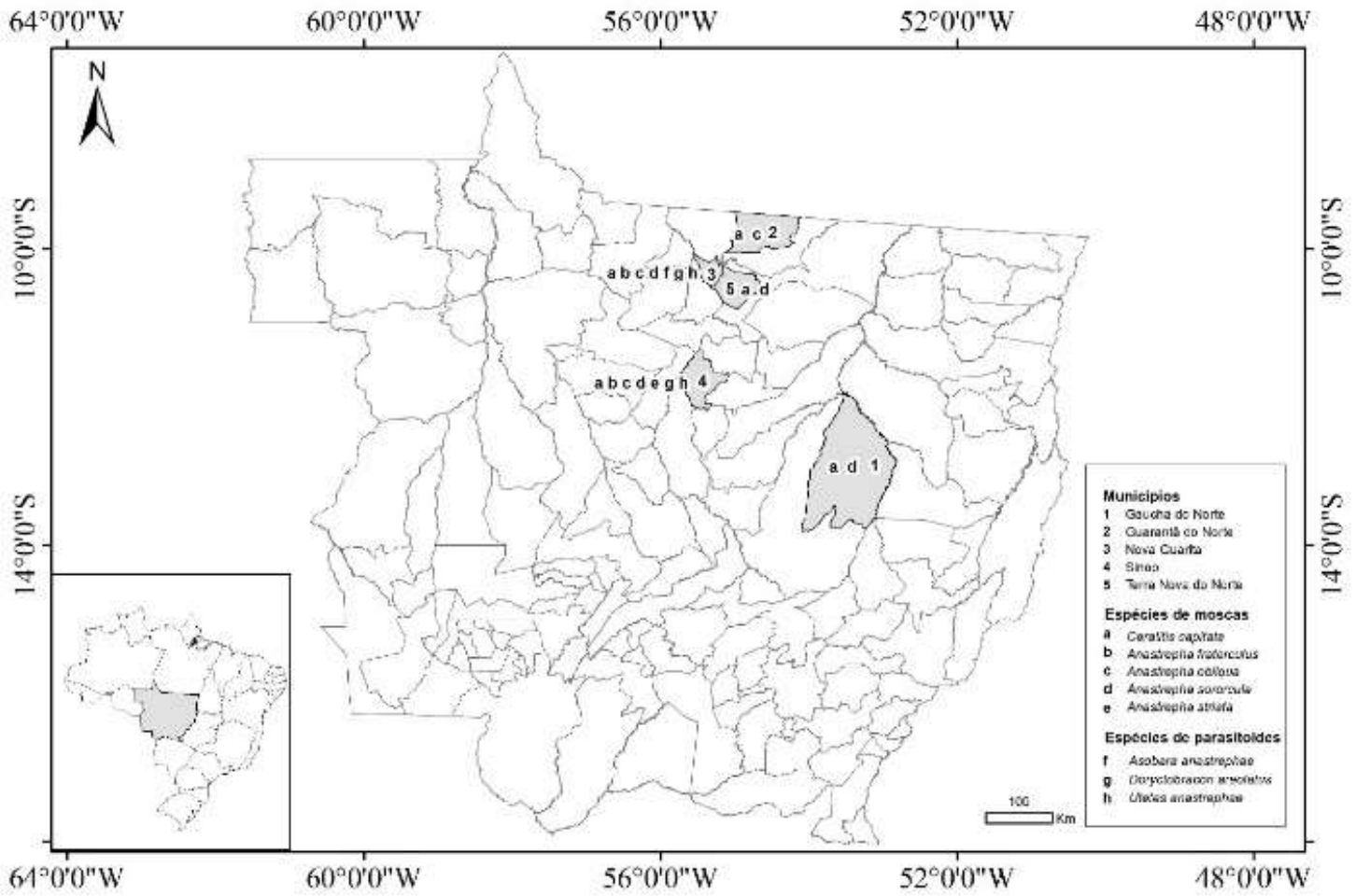

Figura 1. Distribuição geográfica das espécies de moscas-das-frutas e parasitoides no norte do estado de Mato Grosso. Figure 1. Geographic distribution of fruit fly and parasitoid species in the northern state of Mato Grosso.

\section{DISCUSSÃO}

As espécies de moscas-das-frutas obtidas neste estudo já haviam sido reportadas anteriormente no estado do Mato Grosso (UCHOA; PONTES, 2011). Anastrepha obliqua e A. striata ocorrem em todos os Estados que compõem a Amazônia Legal, enquanto que C. capitata é registrada em 7 estados da região amazônica, com exceção do Amapá e Amazonas (ADAIME et al., 2016; ADAIME et al., 2017).

Os primeiros registros de moscas-das-frutas no Estado foram oriundos de coletas pontuais conduzidas na região. Até o ano 2000, somente 9 espécies do gênero Anastrepha estavam reportadas para o Mato Grosso (UCHOA; ZUCCHI, 2000; RONCHI-TELES, 2000). O conhecimento das espécies de tefritídeos foi ampliado com a realização de levantamento em seis municípios do Estado utilizando armadilhas com atrativo alimentar, conduzido por Pontes (2006). Com a descrição de nova espécie por Norrbom; Uchoa, (2011) e com a recente realização de levantamento de espécies com armadilhas no município de Santo Antônio de Leverger, por Silva et al. (2017), atualmente 22 espécies de Anastrepha e C. capitata são registradas no Mato Grosso.

$\mathrm{O}$ registro inicial de hospedeiro de moscas-das-frutas no Mato Grosso foi realizado recentemente por Wochner et al. (2017). A partir de amostras de frutos de Psidium cattleianum Afzel. Ex Sabine (Myrtaceae), coletadas no município de Sinop, em agosto de 2016, foram obtidos espécimes de $A$. striata. Devido à utilização de armadilhas como metodologia para captura de espécimes de moscas-das-frutas, nas coletas realizadas anteriormente no Estado, não havia registros de espécies hospedeiras de tefritídeos e consequentemente associação de espécies de moscas e suas plantas hospedeiras.
Para as espécies de Anastrepha obtidas neste estudo ( $A$. fraterculus, A. obliqua, A. sororcula e A. striata) foram registradas 11 espécies vegetais hospedeiras, a maioria representantes da família Myrtaceae (Tabela 2). Somente $A$. striata possuía registro de hospedeiro (WOCHNER et al., 2017).

O registro do quantitativo de hospedeiros de moscas-dasfrutas é relativamente pequeno na Amazônia, quando comparado a outras regiões do país, considerando-se a diversidade de espécies frutíferas existentes na região (ZUCCHI et al., 2011). As espécies A. obliqua e A. striata são as que possuem o maior número de hospedeiros conhecidos na Amazônia brasileira (ADAIME et al., 2016).

Para $C$. capitata foram registradas 8 espécies de plantas hospedeiras, 4 destas pertencentes à família Myrtaceae, sendo as demais representantes das famílias Anacardiaceae, Malpighiaceae e Oxalidaceae (Tabela 2). Segundo Castilho et al. (2019), na Amazônia brasileira, foram registradas sete espécies vegetais hospedeiras de $C$. capitata, pertencentes a seis famílias. Goiaba e carambola são as mais frequentes, registradas em cinco e quatro Estados, respectivamente. $\mathrm{O}$ Pará é o estado que apresenta o maior número de espécies hospedeiras registradas até o momento (seis).

$\mathrm{Na}$ Amazônia brasileira, até o momento, foram registradas sete espécies vegetais hospedeiras de C. capitata, pertencentes a seis famílias (Tabela 2). Goiaba e carambola são as mais frequentes, registradas em cinco e quatro Estados, respectivamente. $\mathrm{O}$ Pará é o estado que apresenta o maior número de espécies hospedeiras registradas até o momento (seis).

Na Amazônia brasileira, estão assinalados o registro de 8 espécies de parasitoides pertencentes à família Braconidae e 3 
espécies da família Figitidae (ADAIME et al., 2018). Para o Mato Grosso, particularmente, o único registro de parasitoide de moscas-das-frutas foi realizado recentemente por Wochner et al. (2017). Os autores reportaram a ocorrência do parasitoide D. areolatus associado a $A$. striata em frutos de P. cattleianum (Myrtaceae), oriundos de amostras coletadas no município de Sinop (Tabela 3). Porém, com a realização do presente trabalho foi possível ampliar o quantitativo de espécies de parasitoides no Estado, pois foram reportadas pela primeira vez as espécies $U$. anastrephae e $A$. anastrephae.

Observa-se que $D$. areolatus vem se revelando um importante parasitoide no Brasil e na América Latina, tanto por sua abundância como por sua ampla distribuição, sendo, juntamente com Opius bellus Gahan, as espécies predominantes na Amazônia brasileira (CANAL; ZUCCHI, 2000; CARVALHO et al., 2010; OVRUSKI et al., 2000; ADAIME et al., 2018).

Tabela 2. Espécies de moscas-das-frutas (Tephritidae) e suas plantas hospedeiras reportadas para o estado do Mato Grosso, Brasil. Table 2. Species of fruit flies (Tephritidae) and their host plants reported for the state of Mato Grosso, Brazil.

\begin{tabular}{|c|c|c|}
\hline Espécies & Hospedeiros / Nomes científicos & Coordenadas \\
\hline \multirow[t]{2}{*}{ Anastrepha fraterculus (Wiedemann) } & Averrhoa carambola & $10^{\circ} 18^{\prime} 22.388^{\prime \prime} \mathrm{S}-5^{\circ} 24^{\prime} 10.534^{\prime \prime} \mathrm{O}$ \\
\hline & Spondias purpurea & $11^{\circ} 53^{\prime} 40.916^{\prime \prime} \mathrm{S}-55^{\circ} 38^{\prime} 36.78^{\prime \prime} \mathrm{O}$ \\
\hline \multirow[t]{5}{*}{ Anastrepha obliqua (Macquart) } & Averrhoa carambola & $10^{\circ} 18^{\prime} 22.388^{\prime \prime} \mathrm{S}-5^{\circ} 24^{\prime} 10.534^{\prime \prime} \mathrm{O}$ \\
\hline & Spondias purpurea & $\begin{array}{c}9^{\circ} 57^{\prime} 44.0^{\prime \prime} \mathrm{S}-54^{\circ} 53^{\prime} 24.8^{\prime \prime} \mathrm{O} \\
11^{\circ} 53^{\prime} 40.916^{\prime \prime} \mathrm{S}-55^{\circ} 38^{\prime} 36.78^{\prime \prime} \mathrm{O}\end{array}$ \\
\hline & Psidium cattleianum & $11^{\circ} 51^{\prime} 45.81^{\prime \prime} \mathrm{S}-55^{\circ} 22^{\prime} 46.114^{\prime \prime} \mathrm{O}$ \\
\hline & Anacardium occidentale & $11^{\circ} 51^{\prime} 35.759^{\prime \prime} \mathrm{S}-55^{\circ} 27^{\prime} 18.288^{\prime \prime} \mathrm{O}$ \\
\hline & Mangifera indica & $11^{\circ} 51^{\prime} 45.81^{\prime \prime} \mathrm{S}-55^{\circ} 22^{\prime} 46.114^{\prime \prime} \mathrm{O}$ \\
\hline \multirow[t]{6}{*}{ Anastrepha sororcula Zucchi } & Malpighia emarginata & $10^{\circ} 35^{\prime} 58.8^{\prime \prime} \mathrm{S}-55^{\circ} 07^{\prime} 08.3^{\prime \prime} \mathrm{O}$ \\
\hline & Psidium cattleianum & $11^{\circ} 51^{\prime} 45.81^{\prime \prime} \mathrm{S}-55^{\circ} 22^{\prime} 46.114^{\prime \prime} \mathrm{O}$ \\
\hline & Plinia cauliflora & $\begin{array}{l}10^{\circ} 18^{\prime} 21.863^{\prime \prime} \mathrm{S}-55^{\circ} 24^{\prime} 9.968^{\prime \prime} \mathrm{O} \\
11^{\circ} 51^{\prime} 45.81^{\prime \prime} \mathrm{S}-55^{\circ} 22^{\prime} 46.114^{\prime \prime} \mathrm{O}\end{array}$ \\
\hline & Syzygium malaccense & $13^{\circ} 6^{\prime} 59.173^{\prime \prime} \mathrm{S}-53^{\circ} 18^{\prime} 23.911^{\prime \prime} \mathrm{O}$ \\
\hline & Eugenia uniflora & $11^{\circ} 51^{\prime} 45.81^{\prime \prime} \mathrm{S}-55^{\circ} 22^{\prime} 46.114^{\prime \prime} \mathrm{O}$ \\
\hline & Eugenia pyriformis. & $11^{\circ} 51^{\prime} 45.81^{\prime \prime} \mathrm{S}-55^{\circ} 22^{\prime} 46.114^{\prime \prime} \mathrm{O}$ \\
\hline \multirow[t]{2}{*}{ Anastrepha striata Schiner } & Psidium cattleianum & $11^{\circ} 51^{\prime} 45.81^{\prime \prime} \mathrm{S}-55^{\circ} 22^{\prime} 46.114^{\prime \prime} \mathrm{O}$ \\
\hline & Psidium guajava & $11^{\circ} 51^{\prime} 45.81^{\prime \prime} \mathrm{S}-55^{\circ} 22^{\prime} 46.114^{\prime \prime} \mathrm{O}$ \\
\hline \multirow[t]{8}{*}{ Ceratitis capitata (Wiedemann) } & Averrhoa carambola & $\begin{array}{c}10^{\circ} 18^{\prime} 22.388^{\prime \prime} \mathrm{S}-5^{\circ} 24^{\prime} 10.534^{\prime \prime} \mathrm{O} \\
11^{\circ} 50^{\prime} 30.746^{\prime \prime} \mathrm{S}-55^{\circ} 32^{\prime} 1.442^{\prime \prime} \mathrm{O} \\
9^{\circ} 57^{\prime} 44.0^{\prime \prime} \mathrm{S}-54^{\circ} 53^{\prime} 24.8^{\prime \prime} \mathrm{O}\end{array}$ \\
\hline & Spondias purpurea & $11^{\circ} 53^{\prime} 40.916^{\prime \prime} \mathrm{S}-55^{\circ} 38^{\prime} 36.78^{\prime \prime} \mathrm{O}$ \\
\hline & Malpighia emarginata & $\begin{array}{c}13^{\circ} 6^{\prime} 59.173^{\prime \prime} \mathrm{S}-53^{\circ} 18^{\prime} 23.911^{\prime \prime} \mathrm{O} \\
10^{\circ} 18^{\prime} 21.863^{\prime \prime} \mathrm{S}-55^{\circ} 24^{\prime} 9.968^{\prime \prime} \mathrm{O} \\
10^{\circ} 35^{\prime} 55.32^{\prime \prime} \mathrm{S}-55^{\circ} 6^{\prime} 53.798^{\prime \prime} \mathrm{O} \\
10^{\circ} 35^{\prime} 58.8^{\prime \prime} \mathrm{S}-55^{\circ} 07^{\prime} 08.3^{\prime \prime O} \\
11^{\circ} 51^{\prime} 45.81^{\prime \prime} \mathrm{S}-55^{\circ} 22^{\prime} 46.114^{\prime \prime} \mathrm{O} \\
11^{\circ} 50^{\prime} 21.602^{\prime \prime} \mathrm{S}-55^{\circ} 29^{\prime} 54.481^{\prime \prime} \mathrm{O}\end{array}$ \\
\hline & Eugenia stipitata & $10^{\circ} 18^{\prime} 21.863^{\prime \prime} \mathrm{S}-55^{\circ} 24^{\prime} 9.968^{\prime \prime} \mathrm{O}$ \\
\hline & Anacardium occidentale & $11^{\circ} 51^{\prime} 35.759^{\prime \prime} \mathrm{S}-55^{\circ} 27^{\prime} 18.288^{\prime \prime} \mathrm{O}$ \\
\hline & Psidium guajava & $11^{\circ} 51^{\prime} 45.81^{\prime \prime} \mathrm{S}-55^{\circ} 22^{\prime} 46.114^{\prime \prime} \mathrm{O}$ \\
\hline & Plinia cauliflora & $11^{\circ} 51^{\prime} 35.759^{\prime \prime} \mathrm{S}-55^{\circ} 27^{\prime} 18.288^{\prime \prime} \mathrm{O}$ \\
\hline & Eugenia uniflora & $11^{\circ} 51^{\prime} 45.81^{\prime \prime} \mathrm{S}-55^{\circ} 22^{\prime} 46.114^{\prime \prime} \mathrm{O}$ \\
\hline
\end{tabular}

Tabela 3. Espécies de parasitoides (Hymenoptera: Braconidae) de moscas-das-frutas (Tephritidae) reportados no norte estado do Mato Grosso, Brasil. Table 3. Species of parasitoids (Hymenoptera: Braconidae) of fruit flies (Tephritidae) reported in the northern state of Mato Grosso, Brazil.

\begin{tabular}{|c|c|c|c|}
\hline Espécies de parasitoides $^{1}$ & Espécies de moscas ${ }^{1}$ & Plantas hospedeiras $^{1}$ & Coordenadas \\
\hline Asobara anastrephae (Muesebeck) & - & Plinia cauliflora & $10^{\circ} 18^{\prime} 21.863^{\prime \prime} \mathrm{S}-55^{\circ} 24^{\prime} 9.968^{\prime \prime} \mathrm{O}$ \\
\hline \multirow[t]{6}{*}{ Doryctobracon areolatus (Szépligeti) } & - & Plinia cauliflora & $\begin{array}{c}10^{\circ} 18^{\prime} 21.863^{\prime \prime} \mathrm{S}-55^{\circ} 24^{\prime} 9.968^{\prime \prime} \mathrm{O} \\
10^{\circ} 18^{\prime} 50.296^{\prime \prime} \mathrm{S}-55^{\circ} 24^{\prime} 33.455^{\prime \prime} \mathrm{O}\end{array}$ \\
\hline & Anastrepha striata & Psidium cattleianum & $11^{\circ} 51^{\prime} 45.81^{\prime \prime} \mathrm{S}-55^{\circ} 22^{\prime} 46.114^{\prime \prime} \mathrm{O}$ \\
\hline & Ceratitis capitata & Averrhoa carambola & $11^{\circ} 50^{\prime} 30.746^{\prime \prime} \mathrm{S}-55^{\circ} 32^{\prime} 1.442^{\prime \prime} \mathrm{O}$ \\
\hline & Anastrepha striata & Psidium guajava & $11^{\circ} 51^{\prime} 45.81^{\prime \prime} \mathrm{S}-55^{\circ} 22^{\prime} 46.114^{\prime \prime} \mathrm{O}$ \\
\hline & - & Spondias purpurea. & $11^{\circ} 53^{\prime} 40.916^{\prime \prime} \mathrm{S}-55^{\circ} 38^{\prime} 36.78^{\prime \prime} \mathrm{O}$ \\
\hline & - & Eugenia pyriformis & $11^{\circ} 51^{\prime} 56.887^{\prime \prime} \mathrm{S}-55^{\circ} 30^{\prime} 41.116^{\prime \prime} \mathrm{O}$ \\
\hline \multirow[t]{4}{*}{ Utetes anastrephae (Viereck) } & - & Averrhoa carambola & $10^{\circ} 18^{\prime} 22.388^{\prime \prime} \mathrm{S}-55^{\circ} 24^{\prime} 10.534^{\prime \prime} \mathrm{O}$ \\
\hline & - & Plinia cauliflora & $\begin{array}{r}10^{\circ} 18^{\prime} 21.863^{\prime \prime} \mathrm{S}-55^{\circ} 24^{\prime} 9.968^{\prime \prime} \mathrm{O} \\
10^{\circ} 18^{\prime} 50.296^{\prime \prime} \mathrm{S}-55^{\circ} 24^{\prime} 33.455^{\prime \prime} \mathrm{O}\end{array}$ \\
\hline & Anastrepha obliqua & Spondias purpurea & $11^{\circ} 53^{\prime} 40.916^{\prime \prime} \mathrm{S}-55^{\circ} 38^{\prime} 36.78^{\prime \prime} \mathrm{O}$ \\
\hline & - & Eugenia pyriformis & $11^{\circ} 51^{\prime} 56.887^{\prime \prime} \mathrm{S}-55^{\circ} 30^{\prime} 41.116^{\prime \prime} \mathrm{O}$ \\
\hline
\end{tabular}

${ }^{1} \mathrm{~A}$ relação tritrófica (parasitoide/moscas-das-frutas/planta hospedeira) foi considerada devido à emergência de indivíduos de uma única espécie de parasitoide e de mosca na amostra. 


\section{CONCLUSÕES}

Neste trabalho foram apresentados os primeiros registros de plantas hospedeiras de moscas-das-frutas para o Mato Grosso. Por sua vez, os parasitoides Utetes anastrephae e Asobara anastrephae são reportados pela primeira vez no Estado.

\section{AGRADECIMENTOS}

À Júlia Daniela Braga Pereira, pela contribuição a este manuscrito. A Tamara Zamadei pela elaboração do mapa. Ao Conselho Nacional de Desenvolvimento Científico e Tecnológico - $\mathrm{CNPq}$, pela Bolsa de Produtividade em Pesquisa concedida a Ricardo Adaime.

\section{REFERÊNCIAS}

ADAIME, R.; LIMA, A. L.; SOUSA, M. S. M. Controle biológico conservativo de moscas-das-frutas na Amazônia brasileira. Innovations Agronomiques, v. 64, p. 47-59. 2018.

DOI: https://dx.doi.org/10.15454/1.5408013763952266E12

ADAIME, R.; SANTOS, R. S.; SILVA AZEVEDO, T.; SILVA VASCONCELOS, A.; SOUSA, M. D. S. M.; SOUZA-FILHO, M. F. First record of Ceratitis capitata (Wiedemann) (Diptera: Tephritidae) in the state of Acre, Brazil. EntomoBrasilis, Vassouras, v. 10, p. 259-260, 2017.

DOI:

https://dx.doi.org/10.12741/ebrasilis.v10i3.724

ADAIME, R.; SOUSA, M.S.M; PEREIRA, J.F. 2016. Anastrepha species and their hosts in the Brazilian Amazon. In: Meeting of the Tephritid Workers of the Western Hemisphere, 9., 2016, Buenos Aires. Book of Abstracts... Buenos Aires: FAO - AIEA, 2016. p. 146. Disponível em <http://anastrepha.cpafap.embrapa.br> Acesso em 18 out 2018.

ALUJA M.; SIVINSKI J.; VAN DRIESCHE R.; ANZURESDADDA A.; GUILLÉN L. Pest management through tropical tree conservation. Biodiversity and Conservation, London, v. 23, n. 4, p. 831-853, 2014. DOI: https://dx.doi.org/10.1007/s10531-014-0636-3

ALUJA, M.; MANGAN, R. L. Fruit fly (Diptera: Tephritidae) host status determination: critical conceptual, methodological, and regulatory considerations. Annual Review of Entomology, v. 53, p. 473-502, 2008. DOI https://dx.doi.org/10.1146/annurev.ento.53.103106.09335 0

ALVARES, C. A.; STAPE, J. L.; SENTELHAS, P. C.; GONÇALVES, J. L. M.; Köppen's climate classification map for Brazil. Meteorologische Zeitschrift, Berlin, v. $22, \quad$ n. 6, p. 711-728, 2013. DOI: https://dx.doi.org/10.1127/0941-2948/2013/0507

ÁLVARES, V. S.; BAYMA, M. M. A. Evolução na produção de frutas na Amazônia. 2017. Disponível em: $<$ http://www.diadecampo.com.br/zpublisher/materias/Mat eria.asp? $\mathrm{id}=34129 \&$ secao $=$ Artigos $\% 20$ Especiais $>$ Acesso em: 5 ago 2009.

CAMARGOS, M. G.; COSTA, M. L. Z.; MIRANDA, E. S. Custos variáveis de produção de Diachasmimorpha longicaudata (Ashmead) para controle de moscas-dasfrutas. Revista iPecege, Piracicaba, v. 3, n. 2, p. 9-25, 2017. DOI: https://dx.doi.org/10.22167/r.ipecege.2017.2.9
CANAL, D. N. A.; ZUCCHI, R. A. Parasitoide - Braconidae. In: MALAVASI, A.; ZUCCHI, R. A. (Eds.). Moscas-dasfrutas de importância econômica no Brasil: conhecimento básico e aplicado. Ribeirão Preto: Holos, 2000. p. 119-126.

CARVALHO, R. S.; SOARES FILHO, W. S.; RITZINGER, R. Umbu-cajá como repositório natural de parasitoide nativo de moscas-das-frutas. Pesquisa Agropecuária Brasileira, Brasília, v. 45, n. 10, p. 1222-1225. 2010. http://dx.doi.org/10.1590/S0100-204X2010001000024

CASTILHO, A. P.; CORREAA BRANDÃO, C. A.; AYRES, Á. R.; PEREIRA, J. F.; ADAIME, R. Distribuição geográfica e plantas hospedeiras de Ceratitis capitata (Wiedemann) (Diptera: Tephritidae) na amazônia brasileira. In: Jasper, M. (Org.). Coletânea nacional sobre entomologia [recurso eletrônico] / Organizadora - Ponta Grossa, PR: Atena Editora, 2019. p. 90 - 102. DOI 10.22533/at.ed.5041909078

IBGE INSTITUTO BRASILEIRO DE GEOGRAFIA E ESTATÍSTICA. Estabelecimentos agrícolas. 2006. $<$ https://www.ibge.gov.br/estatisticasnovoportal/economicas/agricultura-e-pecuaria/9827censo-agropecuario.html $?=\& \mathrm{t}=$ resultados $>$ Acesso 28 agosto 2018

JESUS-BARROS, C. R.; ADAIME, R.; OLIVEIRA, M. N.; SILVA, W. R.; COSTA-NETO, S. V.; SOUZA-FILHO, M. F. Anastrepha (Diptera: Tephritidae) Species, Their Hosts and Parasitoids (Hymenoptera: Braconidae) in Five Municipalities ofthe State of Amapá, Brazil. Florida Entomologist, v. 95, n. 3, p. 694-705, 2012. DOI: https://dx.doi.org/10.1653/024.095.0320

LORENZI, H.; BACHER, L.; LACERDA, M.; SARTORI, S. Frutas brasileiras e exóticas cultivadas: de consumo in natura. São Paulo: Instituto Plantarum de Estudos da Flora, 2006. $627 \mathrm{p}$

MARINHO, C. F.; SILVA, R. A., ZUCCHI, R. A. Chave de identificação de Braconidae (Alysiinae e Opiinae) parasitoides de larvas frugívoras na região Amazônica. In: SILVA, R. A.; LEMOS, W. P.; ZUCCHI, R. A. (Eds.). Moscas-das-frutas na Amazônia brasileira: diversidade, hospedeiros e inimigos naturais. Macapá: Embrapa, 2011. p. 91-101.

NORRBOM, A. L. \& UCHOA, M. A. New species and records of Anastrepha (Diptera: Tephritidae) from Brazil. Zootaxa, v. 2835 , n. 1, p. 61-67, 2011. DOI: http://dx.doi.org/10.11646/zootaxa.2835.1.5

OVRUSKI, S. M.; ALUJA, M.; SIVINSKI, J.; WHARTON, R. A. Hymenopteran parasitoids on fruit-infesting Tephritidae (Diptera) in Latin America and the Southern United States: diversity, distribution, taxonomic status and their use in fruit fly biological control. Integrated Pest Management Reviews, v. 5, n. 2, p. 81-107. 2000. DOI: https://dx.doi.org/10.1023/A:1009652431251

PEAF MT_Plano Estadual da Agricultura Familiar. Secretaria de Estado Agricultura Familiar e Assuntos Fundiários - MT, 2017. Disponível em $<$ http://www.seaf.mt.gov.br/documents/195721/8110755/ PEAF+-

+PLANO+ESTADUAL+DA+AGRICULTURA+FAMIL IAR+DE+MATO+GROSSO.pdf/3a008fb2-be45-48b11dfb-0ead36a31475>. Acesso 28 agosto 2018 
PONTES, A. V. Biodiversidade de moscas frugívoras (Diptera: Tephritoidea) amostradas com armadilhas McPhail no sudeste de Mato Grosso, Brasil. 2006. 36f. Dissertação (Mestrado em Entomologia e Conservação da Biodiversidade) - Faculdade de Ciências Biológicas e Ambientais, Universidade Federal da Grande Dourados, Dourados, 2006.

RONCHI-TELES B. Ocorrência e flutuação populacional de espécies de moscas-das-frutas e parasitoides com ênfase para o gênero Anastrepha (Diptera: Tephritidae) na Amazônia Brasileira. 2000. 156f. Tese (Doutorado em Ciências Biológicas) - Instituto Nacional de Pesquisas da Amazônia, Universidade do Amazonas, Manaus, 2000.

SILVA, P. S.; SILVA, C. O.; MARTINS, R. S.; MULTANI, J. S. Primeiro registro de Anastrepha fractura e Anastrepha mucronota (Diptera: Tephritidae) no estado do Mato Grosso. Revista Científica Intelletto, Venda Nova do Imigrante, v. 2, n. 1, p. 1-4. 2017.

SILVA, R. A.; LIMA, A. L.; DEUS, E. G. Controle biológico de moscas-das-frutas na Amazônia: um caminho para o desenvolvimento sustentável da fruticultura. Inclusão Social, Brasília, v. 6, n. 2, p. 90-99, 2013.

UCHOA, M. A.; ZUCCHI, R. A. Moscas-das-Frutas nos Estados Brasileiros. Mato Grosso e Mato Grosso do Sul. In: MALAVASI, A.; ZUCCHI, R. A. (Eds) Moscas-dasfrutas de importância econômica no Brasil: conhecimento básico e aplicado. Ribeirão Preto: Holos, 2000. p. 241-245.

UCHOA, M. A.; PONTES, A. V. Species of fruit flies (Diptera: Tephritidae) in the Southeast of Mato Grosso. In: INTERNATIONAL CONGRESS OF DIPTEROLOGY, 7., 2010, San José, Costa Rica. Abstracts... San José: Instituto Nacional de Biodiversidad, n. 253. 2011. Não paginado.

WOCHNER, M. A.; SILVA, M. E. S.; SOUZA, M. S. M.; SILVA, R. A.; BARRETO, M. R. Primeiro registro de parasitismo de Anastrepha striata Schiner (Diptera: Tephritidae) por Doryctobracon areolatus (Szépligeti, 1911) (Hymenoptera: Braconidae) no município de Sinop, Mato Grosso. In: SIMPÓSIO DE CONTROLE BIOLÓGICO, 15., 2017. Anais... Ribeirão Preto, 2017. Não paginado.

ZUCCHI, R. A. Mosca-do-mediterrâneo, Ceratitis capitata (Wiedemann). In: VILELA, E. F.; ZUCCHI, R. A. (Eds.). Pragas introduzidas no Brasil: insetos e ácaros. São Paulo: FEALQ, Piracicaba, 2015. p. 153-172.

ZUCCHI, R. A.; SILVA, R. A.; DEUS, E. G. Espécies de Anastrepha e seus hospedeiros na Amazônia brasileira. In: SILVA, R. A.; LEMOS, W. P.; ZUCCHI, R. A. Moscasdas-frutas na Amazônia brasileira: diversidade, hospedeiros e inimigos naturais. Macapá: Embrapa Amapá, 2011. p. 51-70. 